\title{
Is deliberate hypotension a safe technique for orthopedic surgery?: a systematic review and meta-analysis of parallel randomized controlled trials
}

\author{
Jia Jiang ${ }^{1}$, Ran Zhou' ${ }^{1}$, Bo Li ${ }^{2}$ and Fushan Xue 3 $^{3^{*}}$
}

\begin{abstract}
Background: Deliberate hypotension has been shown to reduce the intraoperative bleeding and the need for allogeneic blood transfusion, and improve the surgical field, but there is still controversy on its clinical safety. This systematic review was designed to assess the safety and benefits of deliberate hypotension for orthopedic surgery.

Methods: The review met the requirements of the PRISMA guidelines. The Cochrane Central Register of Controlled Trials (CENTRAL), MEDLINE, EMBASE, CINAHL, ISI Web of Science, ScienceDirect, and four Chinese databases (China National Knowledge Infrastructure, Wanfang, vip citation database, and updated version of China Biology Medicine disc from January 1, 2000 to January 1, 2019) were searched. All parallel randomized controlled trials comparing the effects of using deliberate hypotension with not using deliberate hypotension on clinical outcomes of patients undergoing orthopedic surgery were selected. The primary outcome was overall mortality. The secondary outcomes were the intraoperative blood loss, blood transfusion volume, and serious adverse postoperative events.

Results: A total of 30 studies with 36 comparisons (1454 participants) were included in meta-analysis. Two studies with 120 participants reported overall mortality and the result was zero (low-quality evidence). The use of deliberate hypotension reduced the intraoperative blood loss (mean difference, $-376.7 ; 95 \% \mathrm{Cl}-428.1$ to $-325.3 ; R^{2}=94 \%$; 29 studies, 36 comparisons, and 1398 participants; low-quality evidence) and blood transfusion volume (mean difference, $-242.5 ; 95 \% \mathrm{Cl}-302.5$ to $-182.6 ; P^{2}=95 \% ; 13$ studies, 14 comparisons, and 544 participants; low-quality evidence). Six studies with 286 participants reported the occurrence of serious adverse postoperative events and the result was zero (low-quality evidence). Subgroup analyses according to age groups, controlled mean artery pressure levels, types of orthopedic surgeries, different combinations of other blood conservative method, and hypotensive methods mostly did not explain heterogeneity; significant differences were identified in almost all subgroups.

Conclusions: Based on the available evidence, it is still unclear whether or not deliberate hypotension is a safe technique for orthopedic surgery due to limited studies with very small sample size, though it may decrease the intraoperative blood loss and blood transfusion volume irrespective of age groups, controlled mean artery pressure levels, types of surgeries, hypotensive methods, or different combinations of other blood conservation strategies.
\end{abstract}

Trial registration: PROSPERO CRD42016045480.

Keywords: Deliberate hypotension, Orthopedic surgery, Randomized controlled trial, Meta-analysis

\footnotetext{
*Correspondence: xuefushan@aliyun.com; fushanxue@outlook.com

${ }^{3}$ Department of Anesthesiology, Beijing Friendship Hospital, Capital Medical

University, Beijing 100050, China

Full list of author information is available at the end of the article
}

(c) The Author(s). 2019 Open Access This article is distributed under the terms of the Creative Commons Attribution 4.0 International License (http://creativecommons.org/licenses/by/4.0/), which permits unrestricted use, distribution, and reproduction in any medium, provided you give appropriate credit to the original author(s) and the source, provide a link to the Creative Commons license, and indicate if changes were made. The Creative Commons Public Domain Dedication waiver (http://creativecommons.org/publicdomain/zero/1.0/) applies to the data made available in this article, unless otherwise stated. 


\section{Background}

Orthopedic surgery always involves the manipulation of bone marrow, muscle tissue, and some venous plexus. Due to complex vascularity, bleeding during orthopedic surgery is relatively large and often manifests as diffused bleeding and is not readily controllable by conventional surgical techniques methods, especially when manipulation involves intrabony capillaries [1]. Thus, blood transfusion, in particular the transfusion of red blood cells, is a common practice in orthopedic surgery [2]. In view of potential adverse effects of blood transfusion [3] and an increasing shortage of blood resources, many efforts have been made on the alternatives to transfusion or blood conservation measures to minimize allogeneic blood transfusion, such as acute hypervolemic or normovolemic hemodilution, perioperative blood salvage, use of epoetin alfa to stimulate erythropoiesis, hemostatic agents, deliberate hypotension, and others $[4,5]$.

Deliberate hypotension refers to any technique that decreases intraoperative blood pressure. Various techniques for deliberate hypotension have been used, including controlling venous return (e.g., changing patient position), and pharmacological interventions (for example, the use of volatile anesthetics, intravenous anesthetics, vasodilators, or $\beta$-adrenoceptor antagonists), intrathecal anesthesia, and others. These hypotensive techniques can be used alone, or in combination. The ideal technique should be easy to perform, have a short onset time, an effect that disappears quickly when drug administration is discontinued, a rapid elimination without toxic metabolites, negligible effects on vital organs, and a predictable and dose-dependent effect. It has been shown that the use of deliberate hypotension can shorten the operative time, reduce the risk of tissue edema caused by ligation or electrocautery, and improve myocardial performance by reducing cardiac preload and afterload [4, 6]. A recent retrospective cohort study indicates that deliberate hypotension has a potential ability to minimize length of hospital stay for patients undergoing orthognathic surgery [7]. In 2007, moreover, a meta-analysis provides evidence to support for the use of deliberate hypotension in orthopedic surgery [8]. However, deliberate hypotension has a potential risk of multiple complications. Even within "a safe range of hypotension," brain damage, stroke, and death may still occur [9]. It is reported in the early 1950s that mortality associated with deliberate hypotension is about $0.22 \%$ to $0.34 \%$, and nonfatal complications mainly referred to cerebral, coronary, and renal circulations occur 908 times (about $2.6 \%$ to $3.3 \%$ ) [10]. In the early 1960 s, a mortality of $0.10 \%$ is reported in 9107 patients with deliberate hypotension [11]. Thus, clinical safety of deliberate hypotension has always been a major concern of clinicians, especially for patients with known hypertension, elderly patients, and those requiring special positions during surgery (e.g., beach-chair position and reverse Trendelenburg position). Nevertheless, no systematic review and meta-analysis on the safety of this technique has been conducted until now. Moreover, the benefits of deliberate hypotension for orthopedic surgery have not been updated since 2007. Thus, this systematic review was performed to assess the safety and benefits of deliberate hypotension for orthopedic surgery.

\section{Methods \\ Eligibility criteria}

This systematic review and meta-analysis was conducted following the recommendations of the Cochrane Handbook for Systematic Reviews and Interventions and reported according to the PRISMA statement (www.prisma-statement. org) [12]. The protocol had been registered on the PROSPERO (http://www.crd.york.ac.uk/PROSPERO CRD420160 45480).

All parallel randomized controlled trials (RCTs) comparing the effects of using deliberate hypotension with not using deliberate hypotension for orthopedic surgery on any primary and secondary outcomes were included, irrespective of language or publication status. Observational studies, randomized cross-over trials, prospective cohort studies, and quasi-randomized studies were excluded. All orthopedic surgical participants irrespective of ages, sexes, or anesthetic methods used were included. Spinal surgery performed by neurosurgeons was also included if deliberate hypotension was used during surgery. Patients scheduled for orthognathic surgery or those with a history of neurologic or psychiatric dysfunction, uncontrolled hypertension, ischemic heart diseases, stroke, renal or hepatic dysfunction, severe peripheral vascular diseases, uncorrected hypovolemia, and anemia (hemoglobin level $\leq 110 \mathrm{~g} / \mathrm{dL}$ ) were excluded. The intervention group used deliberate hypotension by any method. For the control group, blood pressure was not specifically controlled. Studies combining deliberate hypotension with any method of hemodilution (hypervolemic or normovolemic), cell salvage, tourniquet, or other pharmacological interventions (use of hemostatic agents such as hemocoagulase, tranexamic acid, etc.) to reduce blood loss were also included if they were applied equally to groups. The primary outcome was overall mortality. The secondary outcomes were intraoperative blood loss, blood transfusion volume, and serious adverse postoperative events. The definition of outcomes was summarized in Additional file 1: Table S1.

\section{Search strategy}

The current issue of the Cochrane Central Register of Controlled Trials (CENTRAL), MEDLINE (Ovid SP), EMBASE (Ovid SP), CINAHL (via EBSCOhost), ISI Web of Science, ScienceDirect (via Elsvier), and four Chinese databases: China National Knowledge Infrastructure (CNKI), Wanfang, 
vip citation database (VIP), and SinoMed (updated version of China Biology Medicine disc) from January 1, 2000 to January 1, 2019, with no date/time, language, and document type limitations, were searched. Subject search terms with the Cochrane highly sensitive strategies for identifying RCTs described in Section 6.4 of the Cochrane Handbook for Systematic Reviews of Interventions [13] were used to search MEDLINE. The MEDLINE search strategy was applied to search other electronic databases. Keywords were collected through experts' opinion, literature review, controlled vocabulary (medical subject headings $=\mathrm{MeSH}$ and Excerpta Medica Tree = EMTREE), and reviewing the primary search results. The BIOSIS databases (http:// www.biosis.org/), SIGLE database (opensigle.inist.fr), and HMIC database (www.ovid.com/site/catalog/ DataBase/ 99.jsp?top $=2 \&$ mid $=3 \&$ bottom $=7 \&$ subsection $=10$ ) for conference proceedings and grey literature were also searched. Websites of www.clinicaltrials.gov and www. controlled-trials.com/ were searched to identify unpublished trials from January 1, 2000 to January 1, 2019. All search strategies developed by assistance of a medical information specialist were reported in Additional file 2. For literature without full text, we planned to email the study author. The reference lists were screened of all eligible trials and reviews identified for further references to additional trials.

\section{Study selection}

All search results were imported into the Endnote Software by two study authors (JJ and ZR) and duplicate records were removed. If uncertainties remained, the corresponding study author was contacted. Then the title and abstracts were independently screened ( $\mathrm{JJ}$ and ZR). Any obviously irrelevant studies were removed. After retrieving the full texts of any potentially relevant studies, their eligibility was carefully determined. Any disagreement between the two review authors was resolved by discussion with other authors (XFS and LB).

\section{Data extraction and management}

Data was independently extracted by two review authors (JJ and ZR) and entered in our prespecified data collection form (Additional file 2: Table S2). For the continuous data, mean, standard deviation (SD), and sample size were extracted; for studies that only reported median and interquartile range (IQR), median was considered as similar as mean and IQR was approximately 1.35 SD [13]. For the dichotomous variables, the number of events occurred and the sample size were extracted. For rare events that might re-occur to a person or several rare events concurring in one person during the study follow-up period (Poisson data), the total number of events in each group and the total number of person-time at risk in each group were extracted; rates related the counts to the amount of time during which they could have happened [13]. Since a fixed time-point ( 28 days) to follow was set, the time-toevent outcome (mortality) was treated as dichotomous data. For the information that was unable to extract from the available report, the original study authors were contacted. Any disagreements in data extraction were resolved by discussion between two review authors, and if necessary, with a third review author (XFS).

\section{Assessment of risk of bias in included studies}

The risk of bias for each eligible study was assessed by using the "Risk of bias" assessment tool and a "Risk of bias" summary figure was generated by using Review Manager 5.3.5 software. Any disagreement on this assessment was resolved by discussion with a third review author (XFS). For assessment of the risk of bias within and across the included studies, the approach provided in the Cochrane Handbook for Systematic Reviews of Interventions was followed to rate them as unclear, low, or high risk study [13]. The criteria of the GRADE system was used to assess the quality of body of evidences associated with all outcomes [14]. Then a "Grade evidence profile" table was developed by using the GRADE software (www.guidelinedevelopment.org) to rate these outcomes as high, moderate, low, or very low quality. The quality of evidence was downgraded by one or two levels when serious or very serious deficiencies were considered in these criteria. Reporting bias was qualitatively assessed by using funnel plot if the result of the primary outcome was from at least ten trials [15].

\section{Measures of treatment effect}

The risk ratio (RR) and 95\% confidence interval (CI) for dichotomous data were used. The mean difference (MD) and $95 \% \mathrm{CI}$ for continuous data were used when the outcomes in all included studies were made on the same scale. For the rare events that might re-occur to a person during the study follow-up period, such counts were treated as rate data. In addition, trial sequential analysis (TSA) was planned to calculate the required information size for primary outcome and one of secondary outcomes (occurrence of serious adverse postoperative events). The calculation was based on the rate of our control group and the statistics with $\alpha$ and $\beta$ error of 0.05 and 0.20 (two-sided test) and RR reduction of $20 \%$, then the calculated sample size was multiplied by the heterogeneity in our result [16]. $P<0.05$ was considered as statistically significant.

For the studies with more than two intervention groups, such as experimental groups with different methods to induce hypotension, or combined with other methods to modulate blood loss (e.g., hemodilution), or one experimental group with two control groups, the 
"shared" group with similar sample size was split and two or more comparisons were created.

The study author of original report was contacted for important missing statistics. If these data still could not be obtained, the available data was used. If no usable data could be extracted from an eligible study, potential implications of missing data were discussed instead of excluding the study from this review. For the participants' missing due to drop-out, if "missing at random," analysis was performed based on the available data; if not, an available case analysis was performed or if necessary, an intention-to-treat (ITT) analysis was planned. If the study did not mention withdrawals, no drop-outs were assumed.

\section{Data synthesis}

Review Manager 5.3.5 software was used to perform the pooled analysis for the outcomes from more than one study. A chi ${ }^{2}$ test with the $I^{2}$ statistic (with statistical significance set at the level of two-tailed 0.10) was used to describe the percentage of the total variance across studies from heterogeneity rather than from chance. When $I^{2}$ is less than $40 \%$, namely there was no statistical heterogeneity among studies, a fixed-effect model was used; otherwise, a random-effect model was used. In case of evidence of significant heterogeneity, results of both fixed-effect and random-effect models were compared to evaluate if the small study effect had an influence on the treatment effect estimate. If an outcome originated from data of only one study, the estimate of effect was calculated from this single study. For the results that could not be analyzed via meta-analysis, only a qualitative systematic review was performed.

\section{Subgroup analysis and investigation of heterogeneity}

Heterogeneity (clinical and methodological) was considered before performing pooled analysis. Subgroup analyses were performed in the presence of statistical heterogeneity $\left(I^{2} \geq 40 \%\right)$ or an indication of clinical heterogeneity [13]; the following subgroups were considered: (a) age groups: younger than 16 (children), 17 to 65 (adults), older than 65 years of age (elderly patients); (b) controlled mean artery pressure (MAP) levels: $\geq 60 \mathrm{mmHg}, 55$ to $60 \mathrm{mmHg}$, and $<55 \mathrm{mmHg}$; meta-regression was planned to assess the relationship between controlled MAP levels and the primary outcome if no less than ten studies reported the primary outcome were included in the review; (c) types of orthopedic surgeries; (d) different combinations of other blood conservative method; and (e) hypotensive methods used.

\section{Sensitivity analysis}

In order to determine the robustness of our metaanalysis, sensitivity analyses by sequentially removing each high risk study was conducted and the remaining data set for the primary outcome was reanalyzed. Additionally, a previous study suggested that the probability of positive results reported in studies of certain languages, such as Chinese, were significantly higher than other languages [17]. Therefore, a "special" sensitivity analysis was planned by excluding Chinese studies to confirm if the Chinese articles affect the results of pooled analysis for the primary outcome.

\section{Results \\ Description of studies}

The results were presented using the PRISMA statement method [18].

\section{Results of the search}

Using Search strategy, a total of 5886 records were identified. They were de-duplicated (1987 removed) in EndNote $\mathrm{X} 5$ and then sent to two researchers (JJ and ZR) for screening. Further, 3847 were excluded during screening as they were irrelevant to our research question or non-RCT. Fifty-two studies were selected for full text assessment using inclusion and exclusion criteria. Four studies [19-22] were further removed as other blood conservative methods were applied unequally to groups; one [23] because of insufficient information to judge whether the two groups were comparable; two [24, 25] because both groups used deliberate hypotension; six [26-31] because of no relevant outcomes; six [32-37] because of failing to meet inclusion criteria or insufficient information to make a judgment; and two $[38,39]$ because of plagiarism suspected. Thus, 31 studies with 1504 participants) were selected in qualitative synthesis [40-70]; among them, seven studies had two comparisons $[45,53,59,61,64,65,70]$ and one study had no data available (the author's contact information was unavailable) [63]. Finally, 30 studies with 36 comparisons (1454 participants) were included in quantitative synthesis (data extraction for meta-analysis). Process of selection of studies has been shown in PRISMA flow diagram [12] (Fig. 1).

Twenty-four comparisons in 20 studies compared the deliberate hypotension versus control (no deliberate hypotension) [42-44, 49, 51-53, 55-61, 63-65, 68-70]; four comparisons in three studies compared deliberate hypotension combined with acute normovolemic hemodilution versus acute normovolemic hemodilution [40, 45, 47]; seven comparisons in seven studies compared deliberate hypotension combined with acute hypervolemic hemodilution versus acute hypervolemic hemodilution [41, 48, 53, 62, 64, 66, 67]; two studies compared deliberate hypotension combined with acute hypervolemic hemodilution and cell salvage versus acute hypervolemic hemodilution combined with cell salvage [46, 50]; one study compared deliberate hypotension combined with cell 


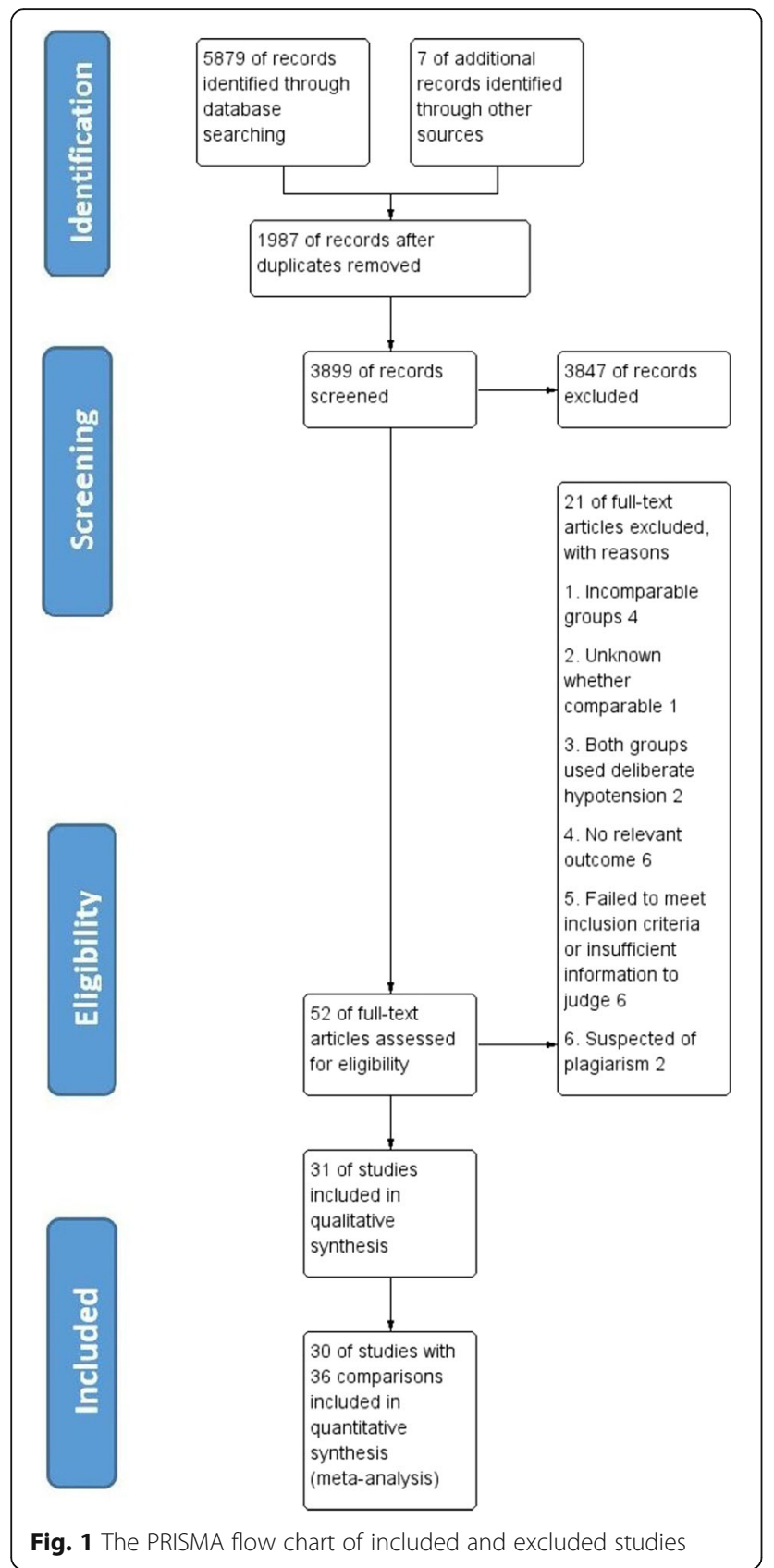

salvage versus cell salvage [54]. Most of the included studies enrolled patients aged 17 to 65 years; four included elderly patients $[43,54-56]$, and seven studies included both adult and elderly patients [44, 46, 49, 50, 52, 58, 62]; four studies did not clearly describe this issue $[60,61,63$, $68]$. The types of surgeries included spine surgery, total hip arthroplasty, pelvic surgery, or femoral fracture surgery. The types of surgeries were not clarified in four studies $[41,42,45,53]$. Eight methods of deliberate hypotension were investigated: remifentanil [40-42, 45, $59,66]$, nitrates [43, 45, 48, 50,51, 53, 55, 59, 61-64], sevoflurane [46, 54], esmolol [47], milrinone [49], epidural anesthesia [58], nicardipine [60, 68, 70], and a combination of two or three hypotensive drugs $[44,52,56,57,61$, $65,67,69,70]$. The controlled MAP level in 14 studies was $\geq 60 \mathrm{mmHg}[40,43,50,54,56,57,60-62,64,66,68$ $70]$; seven studies only limited the percentage of hypotension [44, 45, 51-53, 63, 67]; one study controlled the MAP between 45 and $50 \mathrm{mmHg}$; other studies covered two or more hypotensive level groups. Fourteen studies did not provide the transfusion trigger points [41, 43, $44,46,50,52,55-57,60,63,65,68,69]$. Thirteen studies described the method of measuring the volume of blood loss; most of which was defined as "blood collected in the suction bottles and the increased weight of gauzes" [41, $42,46,48,49,51,53,57,58,60,63,64,67]$. The characteristics of the included studies was summarized in Table 1.

\section{Risk of bias in included studies}

Risk of bias for each study was summarized in Fig. 2 and Additional file 2: Table S3. All included studies except one [49] were classified as unclear or high risk study. The evidence for most of the outcomes was graded as low-quality for imprecision due to very small sample size or for inconsistency due to high level of heterogeneity (Additional file 2: Table S4). The funnel plot indicating publication bias, pre-specified sensitivity analysis, metaregression, and trial sequential analysis for primary outcome could not be done because of limited studies and zero events.

\section{Effects of interventions \\ Primary outcome-overall mortality}

Two studies including 120 participants reported this outcome, one was followed up for $24 \mathrm{~h}$ after surgery [40] and the other was probably followed up during hospital stay [51]; the overall mortality was zero. Seventeen studies including 800 participants although did not explicitly observe the mortality, the occurrence of no death during the observation period could be justified according to pre- and postoperative data of the effects on vital organs, which implied no drop-outs occurred [41, 43, 44, 46, 47, $50,53-56,58,60,65,67-70]$.

\section{Secondary outcomes}

Intraoperative blood loss Thirty studies with 37 comparisons including 1448 participants reported intraoperative blood loss. As one study did not report SD or $P$ value, the data could not be used [63]. Finally, 29 studies with 36 comparisons including 1398 participants were included in pooled analysis [40-46, 48-62, 64-70]. The intraoperative blood loss was reduced by $376.7 \mathrm{ml}$ in the intervention group compared with control group (95\% CI -428.1 to $-325.3 ; P<0.00001 ; I^{2}=94 \%$; Fig. 3). Subgroup analysis according to age groups, controlled MAP 


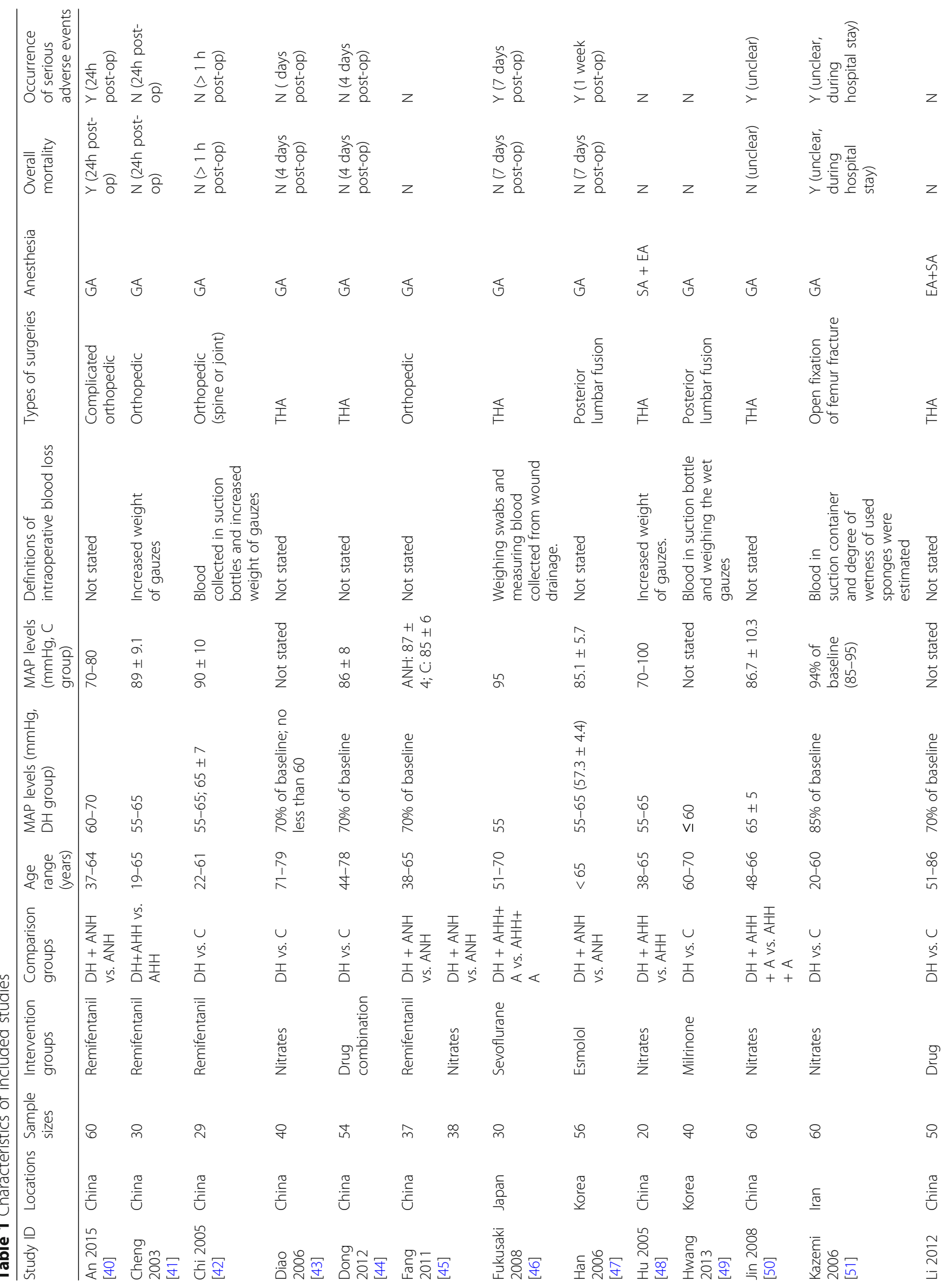




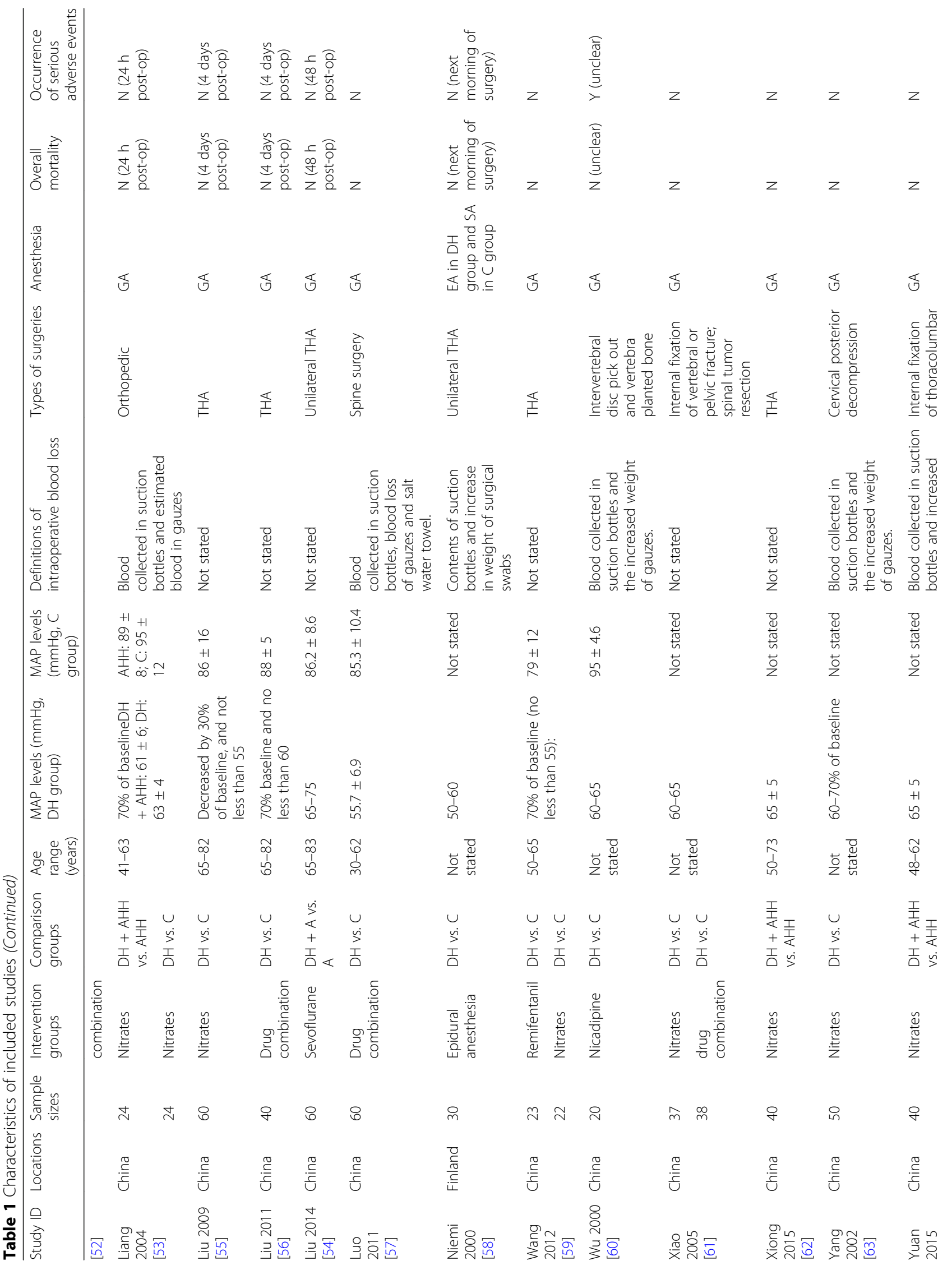




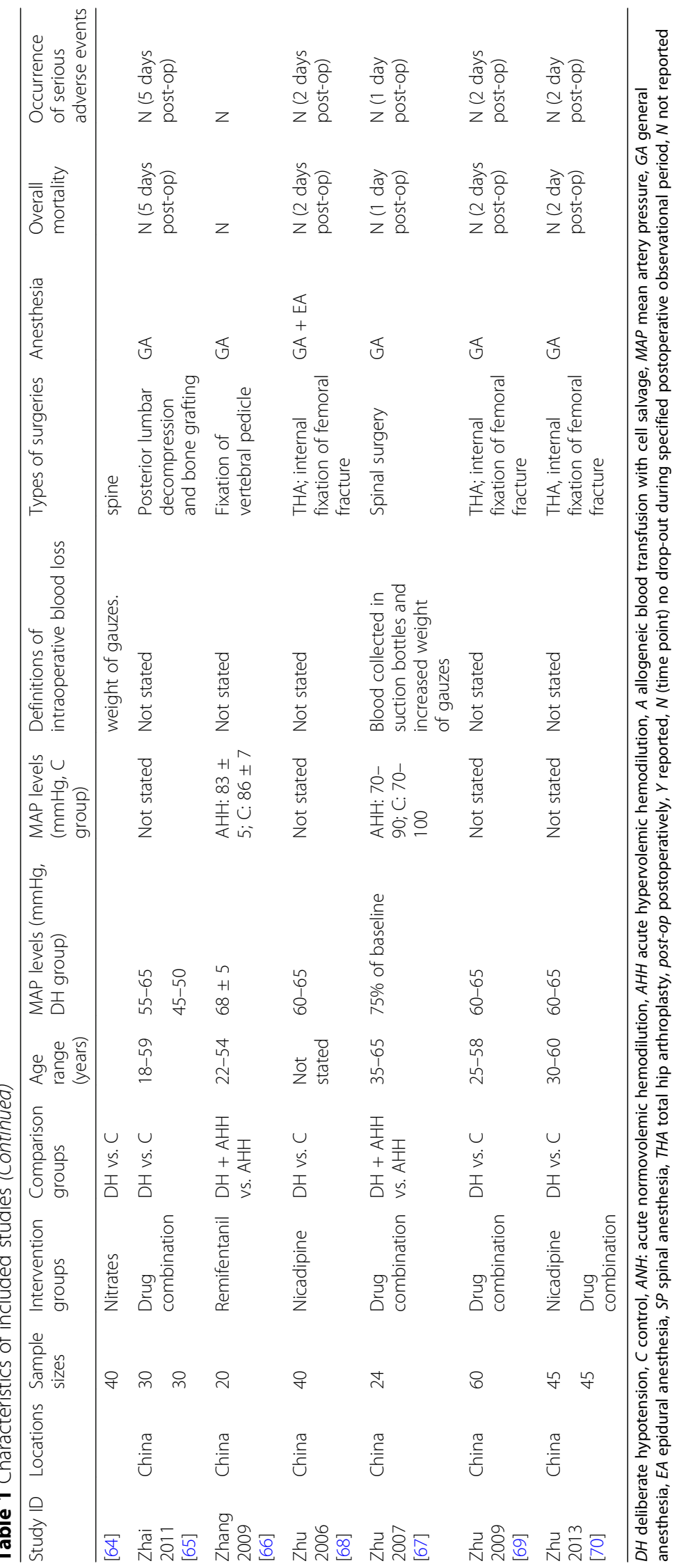




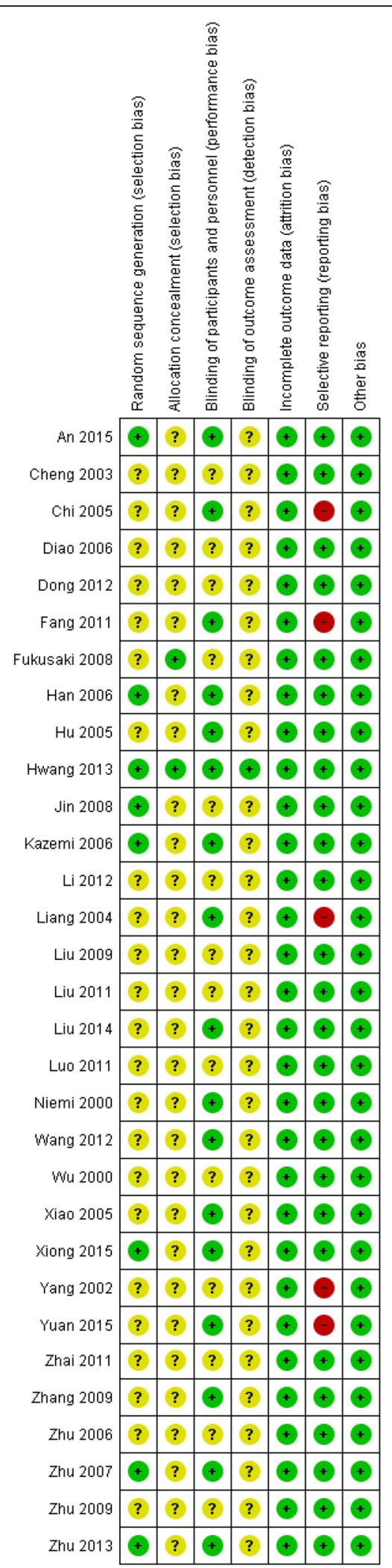

Fig. 2 Risk of bias summary: judgments about each risk of bias item for each included study. Quality evaluation of 31 included studies. +, low risk; -, high risk; ?, unknown levels, types of surgeries, or different combination of other blood conservative methods or hypotensive methods did not explain heterogeneity, except for "elderly" group and deliberate hypotension combined with acute normovolemic hemodilution versus acute normovolemic hemodilution group $\left(I^{2}=0\right)$; and significant differences were identified in all subgroups $(P<0.01)$ except one (deliberate hypotension combined with acute hypervolemic hemodilution and cell salvage versus acute hypervolemic hemodilution combined with cell salvage group); the reason for not reaching significant difference for this subgroup might be the not-overlapped $\mathrm{CI}$ of two included studies in this group; pooling them together might make no sense (Additional file 2: Figures S1-S5).

Blood transfusion volume One study with two comparisons applied acute normovolemic hemodilution, and all the bloods transfused in three groups during surgery were the autologous bloods collected before surgery; no significant differences in the blood transfusion volumes were identified among groups [45]. Five studies reported the number $[51,56,59,64]$ or proportion [53] of patients needing blood transfusion; all of them reported a higher number of patients needing blood transfusion in the control group than in the intervention group, and the data could not be included in meta-analysis. Eighteen studies with 20 comparisons including 754 participants reported blood transfusion volume. The intervention groups in five studies did not receive any type of allogeneic blood transfusions, while all of control groups received allogeneic blood transfusions [48, 50, 54, 58, 70]. Finally, 13 studies with 14 comparisons including 544 participants were included in pooled analysis [40-42, 47, 49, 60, 61, 66-69]. The blood transfusion volume was reduced by $242.5 \mathrm{ml}$ in the intervention group compared with control group (95\% CI -302.5 to $-182.6 ; P<0.01 ; I^{2}=95 \%$; low quality of evidence, Fig. 4). Subgroup analyses according to age groups, controlled MAP levels, types of surgeries, or different combinations of other blood conservative methods or hypotensive methods did not explain heterogeneity, except for "nitrates" group $\left(I^{2}=0\right)$; and significant differences were identified in all subgroups $(P<0.01)$ (Additional file 2 : Figure S6-S10).

Serious adverse postoperative events Six studies including 286 participants reported this outcome, and all results were zero $[40,46,47,50,51,60]$. The durations of followup ranged from $24 \mathrm{~h}$ to 7 days after surgery or during hospital stay. Thirteen studies including 634 participants although did not explicitly observe adverse postoperative events, there was probably the occurrence of no serious adverse postoperative events according to pre- and postoperative indicators (e.g., coagulation index, liver or kidney 


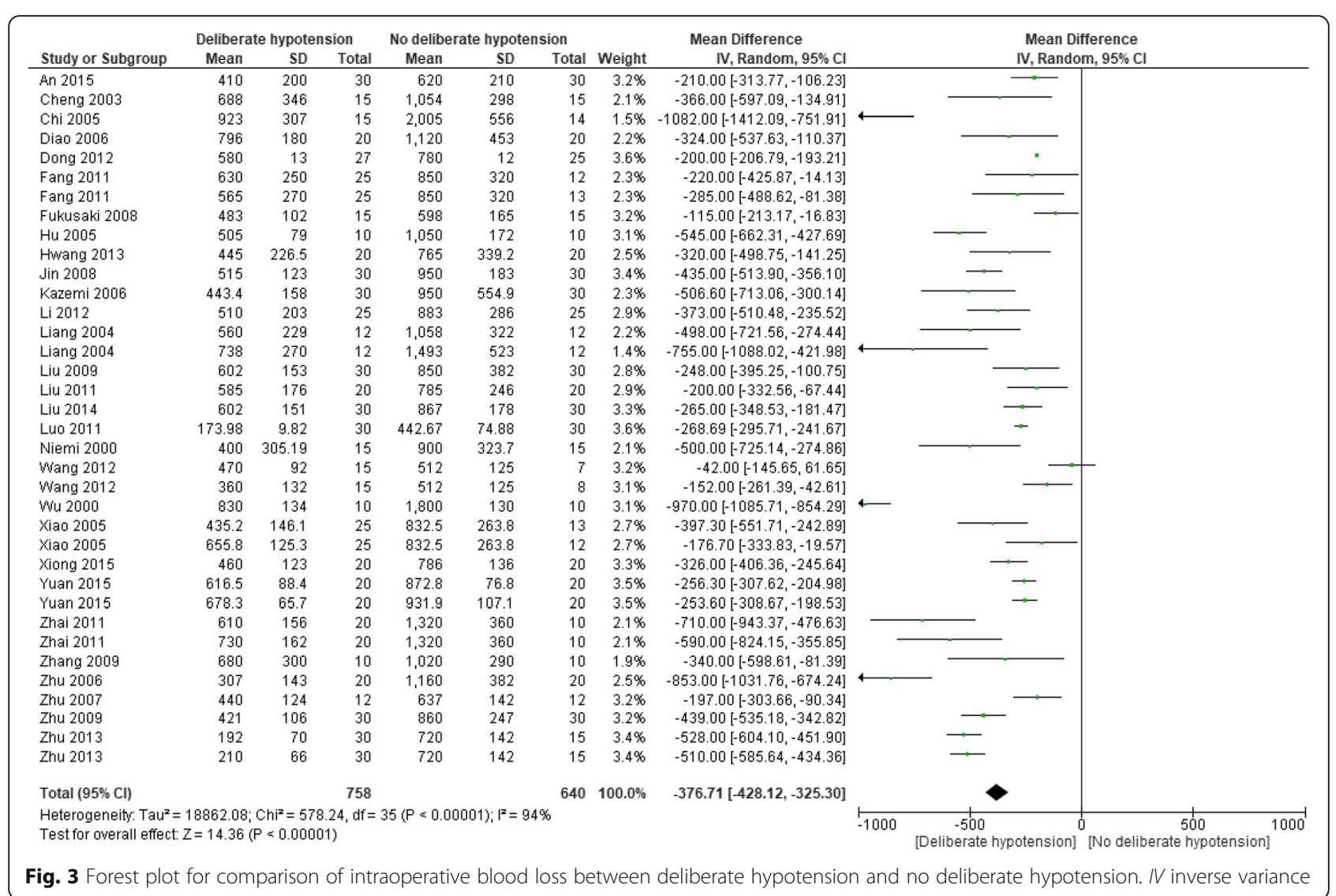

function, cognitive function) or no drop-outs [41, 43, 44, $53-56,58,65,67-70]$.

\section{Discussion}

Our meta-analysis shows the occurrence of no death or serious adverse postoperative events associated with deliberate hypotension in the included studies reporting or implying these outcomes. Furthermore, the use of deliberate hypotension may reduce intraoperative blood loss and blood transfusion volume during orthopedic surgery, irrespective of age groups, controlled MAP levels, types of surgeries, different combinations of other blood conservative methods, or hypotensive methods used.

The safety consideration of using deliberate hypotension in orthopedic surgeries

A systematic review published in 2007 which included patients with orthognathic and orthopedic surgeries and described adverse events associated with deliberate hypotension reported the occurrence of no serious complications or death

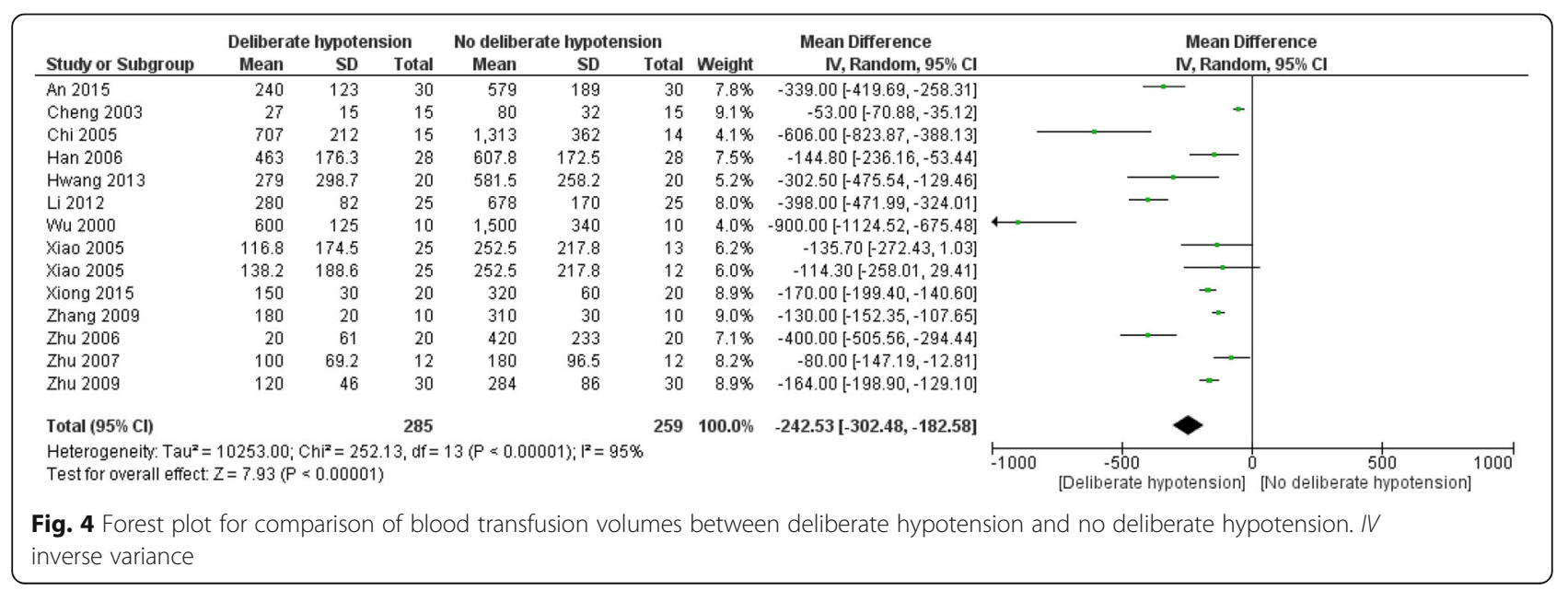


[8]. Orthognathic surgery is excluded in our analysis, as it is quite different from orthopedic surgery in terms of risk of death, intraoperative bleeding, and adverse postoperative events. Pooling the patient with orthognathic and orthopedic surgeries together may introduce significant heterogeneity. A recent observational study including 174 patients with the resection of pelvic and sacral tumors and a potential risk of intraoperative major blood loss assessed the safety of deliberate hypotension, and showed no apparent increase in serious adverse postoperative events and 90-day mortality [71]. A retrospective study examined the use of hypotensive epidural anesthesia in high-risk patients with preoperative renal dysfunction undergoing total hip arthroplasty, and found that hypotension per se, when carefully managed, did not predispose patients with chronic renal dysfunction to acute renal failure after surgery [72].

However, it is still unclear whether or not the use of deliberate hypotension is really safe, as there have been only very few studies reporting mortality and the occurrence of serious adverse postoperative events associated with deliberate hypotension. It has been shown that during the 1950s to 1960 s, mortality associated with deliberate hypotension is $0.10 \%$ to $0.34 \%[10,11]$. With the introduction of new hypotensive agents or methods, generalization of advanced monitoring techniques, and combined use of other blood conservative measures in modern clinical practice, safety of deliberate hypotension would have been further improved. This means that assessment on the adverse outcomes associated with deliberate hypotension may require well designed RCT with an extremely large sample size. Another reason for the occurrence of no serious adverse postoperative events in our included studies may be the enrolment of relatively healthy patients (ASA I and II). In fact, different ages, controlled MAP levels, types of orthopedic surgeries, various combinations of other blood conservative measures, or hypotensive methods used may put patients using deliberate hypotension at different risks of mortality and morbidity. Due to limited number of studies and the occurrence of zero events in available literatures, however, subgroup analyses according to these heterogenetic factors seem impossible.

Although the mortality and serious adverse postoperative events are rare, clinical use of deliberate hypotension still needs caution. In clinical practice, it seems more reasonable to control MAP within a certain percentage of the baseline as to individual conditions of patients, rather than a specific value, especially for patients with hypertension and cardiovascular diseases. Most important, moreover, tissue hypoxia caused by hypotension should not be only limited to the vital organs such as heart, brain, and kidney. A logistic study has shown that hypotension can increase the incidence of postoperative nausea and vomiting [73], which may be related to the imbalance of oxygen supply and demand in the gastrointestinal tissues. Undoubtedly, nausea and vomiting will prolong hospital stay and reduce patients' satisfaction. Another problem is that most of patients undergoing orthopedic surgery are elderly patients with a high risk of postoperative cognitive dysfunction (POCD) [74]. Some studies have observed the effect of deliberate hypotension on POCD [43, 44, 47, 55, 56, 65], but most of them did not use an internationally acknowledged measurement methods, which should include a validated battery of neuropsychological tests to assess global cognitive status, memory, attention, concentration, psychomotor skills, and others [75]. Thus, further studies are needed to observe the effect of deliberate hypotension on the occurrence of POCD in elderly patients undergoing orthopedic surgery.

It is generally believed that combining deliberate hypotension with other blood conservative methods can further reduce allogeneic blood transfusion. In fact, modern blood-sparing strategies also emphasize a combination of various methods. Hypovolemic hemodilution can increase cardiac output [76] and compensate for potential insufficient perfusion caused by decreased blood pressure; cell salvage can reduce allogeneic blood transfusion [50, 54]; tranexamic acid can reduce the bleeding [77]. However, whether combination of these methods with deliberate hypotension is safer than alone use of deliberate hypotension still need further investigation.

\section{Blood-sparing effect of deliberate hypotension}

In previous study [8], our meta-analysis proves that the use of deliberate hypotension may reduce the blood loss and blood transfusion volume during orthopedic surgery. However, there is a high heterogeneity among studies reporting these outcomes. Furthermore, prespecified subgroup analyses mostly do not explain heterogeneity among studies. The experience of surgeons, different transfusion trigger points, various methods of measuring intraoperative blood loss, and within-subgroup heterogeneity (e.g., different types of surgeries) may explain this. Anyway, direction of the effect for almost all subgroups was consistent. From the perspective of sparing blood, deliberate hypotension is still a desirable technique for orthopedic surgery.

\section{Quality of evidence}

The studies included in our analysis were at varying risks of bias and the evidence for outcomes was drawn from RCTs mostly at unclear and high risk of bias. Other than one [49], all of the included studies were justified as "unclear" or "high" risk study, mainly due to no sufficient information on randomization, allocation concealment, and blinding. Although all studies were reported to be randomized, methods of randomization were only described in few studies. Therefore, whether these studies 
have used "real" randomization is still doubtful. Fourteen studies [41, 43, 44, 46, 50, 52, 55-57, 60, 63, 65, 68, 69] did not describe the transfusion trigger points and were not blind to the personnels; this may allow the anesthesiologists to make a decision on blood transfusion only based on personal preference, subjective judgment, or surgeon's demand, resulting in performance bias. Similarly, only 13 studies [41, 42, 46, 48, 49, 51, 53, 57, 58, $60,63,64,67]$ mentioned the details of measuring intraoperative blood loss. Different measurement methods per se can result in a significant heterogeneity among studies; if without any specified measurement method, a detection bias cannot be avoided. In two studies, all of prespecified outcomes were not reported, though these outcomes were not primary or secondary outcomes in our analysis [42, 45]. In three studies, blood transfusion data were reported incompletely, which could not be included in our analysis [53,63,64]. Although a reporting bias may exist in these studies, the final results may probably not be influenced by lacking of these data. Due to limited number of studies included in our analysis, the funnel plot cannot be obtained and a possible publication bias cannot be excluded.

\section{Limitations}

There are some limitations in our analysis. First, subgroup analyses were performed based on possible heterogeneity, but grouping a subgroup was relatively arbitrary, which might have resulted in the heterogeneity within subgroups. Furthermore, experience of surgeons, different transfusion trigger points, various methods of measuring intraoperative blood loss, and within-subgroup heterogeneity (e.g., different types of surgeries) may also introduce heterogeneity. Second, for multiple-comparison studies, the "shared" group was split with similar sample size to create two comparisons. A unit-of-analysis error may occur accordingly, even though this can facilitate investigation of heterogeneity and subgroup analyses. Third, our analysis only included the orthopedic surgeries in supine, lateral, and prone positions. For orthopedic surgeries requiring deliberate hypotension under other special position, such as shoulder arthroscopic surgery with a beach chair position, whether deliberate hypotension will bring additional risk and to what extent MAP level should be controlled are need further studies.

\section{Conclusions}

Based on available evidence, it is still unclear whether or not deliberate hypotension is really safe for orthopedic surgery due to limited studies with very small sample size. However, deliberate hypotension may decrease intraoperative blood loss and blood transfusion volume irrespective of ages, controlled MAP levels, types of surgeries, hypotensive methods, or different combinations of other blood conservation strategies. From the perspective of sparing blood, deliberate hypotension is still a desirable technique for orthopedic surgery. The high-quality evidence from large well-designed RCTs is still needed to clarify the safety of this blood conservation technique for orthopedic surgery.

\section{Supplementary information}

Supplementary information accompanies this paper at https://doi.org/10. 1186/s13018-019-1473-6.

Additional file 1: The search strategy of four electronic databases. Additional file 2: Table S1. Definition of outcomes. Table S2. Data collection form. Table S3. Risk of bias assessment of 31 included RCTs. Table S4. The GRADE for all outcomes.

Additional file 3: Figure S1. Forest plot for comparison of intraoperative blood loss based on different age groups between deliberate hypotension and no deliberate hypotension. IV, Inverse Variance. Figure S2. Forest plot for comparison of intraoperative blood loss based on different controlled MAP levels between deliberate hypotension and no deliberate hypotension. IV, Inverse Variance. Figure S3. Forest plot for comparison of intraoperative blood loss based on types of orthopedic surgeries between deliberate hypotension and no deliberate hypotension. IV, Inverse Variance. Figure S4. Forest plot for comparison of intraoperative blood loss based on different combinations of other blood conservative method between deliberate hypotension and no deliberate hypotension. IV, Inverse Variance; $\mathrm{DH}$ : deliberate hypotension; $\mathrm{C}$ : control; $\mathrm{ANH}$ : acute normovolemic hemodilution; $\mathrm{AHH}$ : acute hypervolemic hemodilution; $A$ : autologous blood transfusion with cell salvage. Figure S5. Forest plot for comparison of intraoperative blood loss based on different hypotensive methods between deliberate hypotension and no deliberate hypotension. IV, Inverse Variance. Figure S6. Forest plot for comparison of blood transfusion volume based on different age groups between deliberate hypotension and no deliberate hypotension. IV, Inverse Variance. Figure S7. Forest plot for comparison of blood transfusion volume based on different controlled MAP levels between deliberate hypotension and no deliberate hypotension. IV, Inverse Variance. Figure S8. Forest plot for comparison of blood transfusion volume based on different types of orthopedic surgeries between deliberate hypotension and no deliberate hypotension. IV, Inverse Variance. Figure S9. Forest plot for comparison of blood transfusion volume based on different combinations of other blood conservative method between deliberate hypotension and no deliberate hypotension. IV, Inverse Variance; DH: deliberate hypotension; C: control; ANH: acute normovolemic hemodilution; $\mathrm{AHH}$ : acute hypervolemic hemodilution; A: autologous blood transfusion with cell salvage. Figure S10. Forest plot for comparison of blood transfusion volume based on different hypotensive methods between deliberate hypotension and no deliberate hypotension. IV, Inverse Variance.

\section{Abbreviations}

Cl: Confidence interval; IQR: Interquartile range; MAP: Mean artery pressure; RCTs: Randomized controlled trials; SD: Standard deviation

\section{Acknowledgments}

We would like to thank Professor Tiansong Zhang for his suggestion on statistical methods used to measure the treatment effect. We also would like to thank Dr. Tie Liu from Department of Orthopedics for his suggestion on inclusion criteria of surgical type and translation of several Japanese articles.

\section{Authors' contributions}

JJ and RZ significantly contributed to the design and implementation of the study, as well as analysis and interpretation, and they drafted the manuscript. BL participated substantially in data acquisition and interpretation. FSX significantly contributed to the conception of the study, performed data analysis and interpretation, and critically revised the manuscript. All authors saw the original study data, reviewed the analysis of the data, and read and approved the final manuscript. 


\section{Funding}

None.

\section{Availability of data and materials}

All data generated or analyzed during this study are included in this published article.

\section{Ethics approval and consent to participate}

This study did not need ethical approval and consent from any patients involved in the study was also not needed.

\section{Consent for publication}

Not applicable.

\section{Competing interests}

The authors declare they have no competing interests.

\section{Author details}

'Department of Anesthesiology, Beijing Chaoyang Hospital, Capital Medical University, Beijing 100020, China. ${ }^{2}$ Beijing Hospital of Traditional Chinese Medicine, Affiliated with Capital Medical University, Beijing Institute of Traditional Chinese Medicine, Beijing 100010, China. ${ }^{3}$ Department of Anesthesiology, Beijing Friendship Hospital, Capital Medical University, Beijing 100050, China.

\section{Received: 8 September 2019 Accepted: 13 November 2019} Published online: 02 December 2019

\section{References}

1. Theusinger OM, Kind SL, Seifert B, Borgeat L, Gerber C, Spahn DR. Patient blood management in orthopaedic surgery: a four-year follow-up of transfusion requirements and blood loss from 2008 to 2011 at the Balgrist University Hospital in Zurich. Switzerland. Blood Transfus. 2014;12(2):195-203.

2. Blood Matters P, Safety, Patient Experience Branch, Victorian Government, Department of Health (2015) Blood management in elective orthopaedic surgery 2009 revised.

3. Isbister JP, Shander A, Spahn DR, Erhard J, Farmer SL, Hofmann A. Adverse blood transfusion outcomes: establishing causation. Transfus Med Rev. 2011; 25(2):89-101.

4. Dragan S, Kulej M, Krawczyk A, Wall A, Plocieniak K, Urbanski W. Methods of reducing allogeneic blood demand in orthopedic surgery. Ortop Traumatol Rehabil. 2012;14(3):199-214.

5. Spahn DR, Goodnough LT. Alternatives to blood transfusion. Lancet. 2013; 381(9880):1855-65.

6. Tagarakis GL, Whitlock RP, Gutsche JT, Diegeler A, Patel PA, Daskalopoulos ME, Vernick WJ, Anastasiadis K, Augoustides JG. New frontiers in aortic therapy: focus on deliberate hypotension during thoracic aortic endovascular interventions. J Cardiothorac Vasc Anesth. 2014;28(3):843-7.

7. Ettinger KS, Yildirim Y, Weingarten TN, Van Ess JM, Viozzi CF, Arce K. Hypotensive anesthesia is associated with shortened length of hospital stay following orthognathic surgery. J Oral Maxillofac Surg. 2015;74(1):130-8.

8. Paul JE, Ling E, Lalonde C, Thabane L. Deliberate hypotension in orthopedic surgery reduces blood loss and transfusion requirements: a meta-analysis of randomized controlled trials. Can J Anesth. 2007:54(10):799-810.

9. Kim JS, Ko SB, Shin HE, Han SR, Lee KS. Perioperative stroke in the brain and spinal cord following an induced hypotension. Yonsei Med J. 2003:44(1):143-5.

10. Little DM. Induced hypotension during anesthesia and surgery. Anesthesiology. 1955;16(3):320-32.

11. Enderby GE. A report on mortality and morbidity following 9,107 hypotensive anaesthetics. Br J Anaesth. 1961;33(2):109-13.

12. Moher D, Liberati A, Tetzlaff J, Altman DG. Preferred reporting items for systematic reviews and meta-analyses: the PRISMA Statement. BMJ. 2009; 3(3):123-30.

13. Higgins JPT, Green S. Cochrane Handbook for Systematic Reviews of Interventions Version 5.1.0 [updated March 2011]. The Cochrane Collaboration, 2011, Available from www.cochrane-handbook.org

14. Guyatt G, Oxman AD, Akl EA, Kunz R, Vist G, Brozek J, Norris S, Falck-Ytter $Y$, Glasziou P, DeBeer H, Jaeschke R, Rind D, Meerpohl J, Dahm P, Schunemann HJ. GRADE guidelines: 1. Introduction-GRADE evidence profiles and summary of findings tables. J Clin Epidemiol. 2011;64(4):383-94.
15. Egger M, Davey SG, Schneider M, Minder C. Bias in meta-analysis detected by a simple, graphical test. BMJ. 1997;315(7109):629-34.

16. Wetterslev J, Thorlund K, Brok J, Gluud C. Estimating required information size by quantifying diversity in random-effects model meta-analyses. BMC Med Res. Methodol. 2009;9:86.

17. Vickers A, Goyal N, Harland R, Rees R. Do certain countries produce only positive results? A systematic review of controlled trials. Control Clin Trials. 1998;19(2):159-66.

18. Guyatt GH, Oxman AD, Kunz R, Vist GE, Falck-Ytter Y, Schunemann HJ. What is "quality of evidence" and why is it important to clinicians. BMJ. 2008; 336(7651):995-8.

19. Cang J, Ma Q, Xue ZG, Jiang H. Effects of deliberated hypotension combined with acute isovolemic haemodilution on extravascular lung water (Chinese). Fudan Univ J Med Sci. 2003;30(4):388-90.

20. Fathi $M$, Jahanbakhsh $\mathrm{S}$, Saadatfar $\mathrm{H}$, Bameshki A, Joudi M. Comparison of aprotinin and controlled hypotension on blood loss in the herniated intervertebral disc surgery. Razavi Int J Med. 2015;3:e29474.

21. Juelsgaard P, Larsen U, Sørensen JV, Madsen F, Søballe K. Hypotensive epidural anesthesia in total knee replacement without tourniquet: reduced blood loss and transfusion. Reg Anesth Pain Med. 2001;26(2): 105-10.

22. Tuncali B, Karci A, Bacakoglu AK, Tuncali BE, Ekin A. Controlled hypotension and minimal inflation pressure: a new approach for pneumatic tourniquet application in upper limb surgery. Anesth Analg. 2003;97(5):1529-32.

23. Chen YS. Application of controlled hypotension in pedicle screw fixation (Chinese). Med J Chin People's Health. 2008;20(9):899.

24. Ikeya K, Kashimoto S, Kumazawa T. Effect of prostaglandin E1 infusion during and after total hip arthroplasty under hypotensive anesthesia on postoperative renal function and urine volume. Japan J Anesthesiol. 2002:51:377-81.

25. Ikeya K, Kume M, Sato H, Kashimoto S, Kumazawa T. Effect of prostaglandin E1 infusion during and after total hip arthroplasty under hypotensive anesthesia on postoperative liver function and hemorrhage. Japan J Anesthesiol. 2003:52(6):616-20.

26. Chen JH, Luo JH. Effects of nitroglycerin-controlled hypotension on cognitive function in elderly patients undergoing hip replacement with sevoflurane anesthesia (Chinese). J Chin Physician. 2012;14(1):76-8.

27. He YJ. Effect of controlled hypotension with urapidil on quality of surgical field in hypertensive patients undergoing clavicular surgery (Chinese). Youjiang Med J. 2012;34(5):629-30.

28. Huo SP. Effects of different hypotension levels on cognitive function after total hip replacement in geriatric patients (Chinese). J Hebei Med Univ. 2012:33(12):1415-7.

29. Huo SP, Li Y, Zhao HY, Wang XL, Wang QJ. Effects of different hypotension levels on renal function after total hip replacement in geriatric patients (Chinese). J Hebei Med Univ. 2013;34(11):1415-8.

30. Ivanova L. Postoperative autologous blood reinfusion in spinal surgery against the background of controlled hypotension. Ortopediya i Travmatologiya. 2000;36:516-9.

31. Peng HH, Liu HY, Fan YL, Huang F, Cheng PR, Jiang WH. Effects of magnesium sulfate on postoperative cognitive dysfunction and cerebral oxygen metabolism during controlled hypotension in elderly patients undergoing hip-replacement (Chinese). Int Med Health Guid News. 2013;19(16):2560-2.

32. Huang $\mathrm{P}$, Huang $\mathrm{ZH}$. Clinical observation of controlled hypotension for total hip arthroplasty (Chinese). Youjiang Med J. 2003;25(6):823-4.

33. Lin HY, Lin J, Yang XX. Effect of controlled hypotension on knee arthroplasty in elderly patients undergoing spinal anaesthesia with ropivacaine (Chinese). Int Med Health Guid News. 2011;17(20):2493-6.

34. Liu L, Liu Y, Wang H, Lu MJ, Zhang JP, Tao Q, Guo JR. Effect of acute normovolemic haemodilution combined with controlled hypotension on extravascular lung water and oxygenation in elderly orthopaedic surgery patients (Chinese). J Clin Anesthesiol. 2017;33(3):213-7.

35. Tan JY, Zhang XD, Guo LD. The effect of epidural controlled hypotension on total blood loss during total hip arthroplasty (Chinese). China Pract Med. 2016;11(30):195-6.

36. Wang SF. The application of controlled hypotension in orthopedic surgery (Chinese). Contemporary Med. 2017;23(4):111-2.

37. Yao MF, Wang QL, Bi JM, Jiang XH, Lu CZ. Effect of nitroglycerin on deep venous thrombosis after total hip arthroplasty (Chinese). J Clin Anesthesiol. 2015;31(7):652-4. 
38. Liu XB. Effect of controlled hypotension with nitroglycerin combined with remifentanil for total hip arthroplasty (Chinese). Henan J Surg. 2015;21(1):68-9.

39. Nong WH. Observation of application of controlled decompression in orthopedics vertebral internal fixation (Chinese). Chin Med Engineering. 2015;23(4):174-5.

40. An XF, Wang XH. Application of remifentanil-hypotension combined with acute normovolemic haemodilution for patients undergoing complicated orthopedic operation (Chinese). Zhejiang Med J. 2015;37(19):1600-3.

41. Cheng MH, Li L, Fang XS, Chen ZQ. Feasibility of application of acute hypervolaemic haemodilution combined with controlled hypotension on patients undergoing orthopedic surgery (Chinese). Chin J Mod Med. 2003; 13(8):70-3.

42. Chi MY, Li FG, Chen QF, Gao XJ, Yu AL, Zhang YW, Zhang ZW, Pan XJ. Observation on the effect of acute hypervolaemic haemodilution combined with controlled hypotension (Chinese). J Chin Physician. 2005;7(10):1412-3.

43. Diao S, Liu PR, Jiang J, Wang GP. Effects of nitroprusside induced hypotension on cognitive function after total hip replacement in geriatric patients (Chinese). Int J Anesth Resus. 2006;27(3):152-4.

44. Dong LP. Effect of remifentanil combined with nitroglycerin in total hip arthroplasty (Chinese). Henan J Surg. 2012;18(6):65-6.

45. Fang JJ, Guo JS, Zhou SY, Jiao L, Liu L, Zhou P, Zhou W. The application of acute normovolemic haemodilution and deliberate hypotension with remifentanil in orthopaedic surgery (Chinese). Contemporary Med. 2011; 17(28):12-4.

46. Fukusaki M, Kanaide M, Inadomi C, Takada M, Terao Y, Sumikawa K. The effect of sevoflurane-induced hypotension in combination with acute hypervolaemic haemodilution on middle cerebral artery flow velocity in surgical patients. Eur J Anaesthesiol. 2008;25(8):657-61.

47. Han SH, Bahk JH, Kim JH, Lim YJ, Park CD, Do SH, et al. The effect of esmolol-induced controlled hypotension in combination with acute normovolemic hemodilution on cerebral oxygenation. Acta Anaesthesiol Scan. 2006:50(7):863-8

48. Hu DN, Qiu WD, Gu YF, Yang L. Clinical study of acute hypervolaemic haemodilution combined with controlled hypotension for total hip arthroplasty (Chinese). J Prac Med. 2005;21(6):585-6.

49. Hwang W, Kim E. The effect of milrinone on induced hypotension in elderly patients during spinal surgery: a randomized controlled trial. Spine J. 2014; 14(8):1532-3.

50. Jin XL, Lv WY, Chen ZB, Lv J, Guo XC. Applicacation of acute hypervolaemic haemodilution combined with hypotension controlled and autologous blood transfusion on patients undergoing total hip replacement (Chinese). $J$ Clin Anesthesiol. 2008:24(7):574-6.

51. Kazemi AP, Zand F, Sedaghat M. Blood-sparing efficiency of transdermal nitroglycerine during open fixation of femur shaft fractures: a randomized, double-blind study (Chinese). J Clin Anesthesiol. 2006;18(7):495-8.

52. Li YH, Liu TX. Application of controlled hypotension with nitroglycerin combined with propofol in total hip arthroplasty (Chinese). Guide Chin Med. 2012;10(21):158-9.

53. Liang SJ, Ma H, Sun HJ, Wang JK. The feasibility of acute hypervolaemic haemodilution combined with controlled hypotension in patients undergoing elective orthopedic operations (Chinese). Chin J Anesthesiol. 2004;24(12):885-8.

54. Liu B, Yao AJ, Feng ZY, Zhu JQ, He ZH. Blood conservative effect of moderate controlled hypotension combined with intraoperative autologous blood transfusion for elderly patients undergoing total hip arthroplasty (Chinese). Prac Clin Med. 2014;30(18):2936-8

55. Liu PR, Diao S, Sun HF, Wang GP, Huang QY. Effects of controlled hypotension with sodium nitroprusside on cognitive function and cerebral oxygen metabolism in elderly patients with hypertension undergoing total hip arthroplasty (Chinese). J Clin Anesthesiol. 2009;25(6):500-1.

56. Liu QS, Zhang ZL, Sun HJ, Liang SJ. Effects of remifentanil combined with nitroglycerin-controlled hypotension on cognitive function in elderly patients undergoing total hip arthroplasty (Chinese). Hebei Med J. 2011; 33(15):2283-4.

57. Luo SH. Observation of application of controlled hypotension in orthopedics vertebral internal fixation (Chinese). J Zhejiang Chin Med Univ 2011;35(3):353-4.

58. Niemi $\Pi$, Pitkanen $M$, Syrjala $M$, Rosenberg PH. Comparison of hypotensive epidural anaesthesia and spinal anaesthesia on blood loss and coagulation during and after total hip arthroplasty. Acta Anaesthesiol Scand. 2000;44(4):457-64.
59. Wang J, Dong SH. Effect of controlled hypotension with remifentanil on renin-angiotensin-aldosterone system of patients undergoing total hip arthroplasty (Chinese). J Chengdu Med Univ. 2012;7(4):601-4.

60. Wu XJ, Hu J, Wang JK. Clinical study of controlled hypotension with nicardipine in orthopedic surgery (Chinese). J China Med Univ. 2000;29(3):230-1.

61. Xiao WP, Zhou QH, Yao M, An ED, Du YF. The clinical observation of controlled-hypotension induced by combination of nitroglycerin and Labetalol in patients undergoing severe orthopedic operations (Chinese). Chin J Exper Surg. 2005;22(12):1571-3.

62. Xiong HP, Wu ZY, Zha BJ. Clinical study of acute hypervolaemic haemodilution combined with controlled hypotension in patients undergoing total hip arthroplasty (Chinese). Clin J Med Officers. 2015; 43(11):1173-7.

63. Yang H. Study of controlled hypotension to reduce bleeding during posterior cervical decompression (Chinese). Qingdao Med J. 2002:34(2):110.

64. Yuan $L B$, Jing $L B$, Jing TP, He YL, Liu XH, Ding ZG, Huang W, Liang H. Blood conservative effect of acute hypervolemic hemodilution combined with controlled hypotension in spinal surgery (Chinese). Med I National Defending Forces in Northwest China. 2015;36(1):4-7.

65. Zhai YY, Liu YZ, Liu YB, Wang SD. Effects of low-level controlled hypotension on postoperative cognitive function in patients undergoing spinal surgery (Chinese). Shandong Med J. 2011;51(49):90-1.

66. Zhang QM, Zhang JG, Yang J. Clinical application of controlled hypotension with remifentanil and propofol combined with blood dilution in pedicle screw fixation (Chinese). Proc Clin Med. 2009;18(10):742-4.

67. Zhu J, Zhao XL, Feng H, Zheng $\mathrm{H}$. Effect of acute hypervolaemic hemodilution combined with controlled hypotension on coagulation function in patients undergoing spinal surgery (Chinese). Xinjiang Med J. 2007;37(6):8-12.

68. Zhu JF. Clinical study of combined general-epidural anaesthesia with controlled hypotension in orthopedic surgery (Chinese). Chin J Prim Med Pharm. 2006;13(1):62-3.

69. Zhu JF, Feng ZM. Clinical study of controlled hypotension with nicardipine combined with Laberol (Chinese). Shanxi Med J. 2009;38(12):1610-1.

70. Zhu JF, Feng ZM. Dexmedetomidine combined with nicardipine for controlled anti-hypertensive therapy in orthopedic surgery (Chinese). Chin J Biomed Engineering. 2013;19(4):317-20.

71. Freeman AK, Thorne CJ, Gaston CL, Shellard R, Neal T, Parry MC, Grimer RJ, Jeys L. Hypotensive Epidural Anesthesia Reduces Blood Loss in Pelvic and Sacral Bone Tumor Resections. Clin Orthop Relat R. 2017;475(3):634-40.

72. Sharrock NE, Beksac B, Flynn E, Go G, Della Valle AG. Hypotensive epidural anaesthesia in patients with preoperative renal dysfunction undergoing total hip replacement. Br J Anaesth. 2006;96(2):207-12.

73. Liu QS, Wu CL. Nausea and vomiting after gynaecological laparoscopy related factors and nursing (Chinese). Chin Primary Health Care. 2010; 24(8):99-100.

74. Kadono Y, Yasunaga H, Horiguchi H, Hashimoto H, Matsuda S, Tanaka S, et al. Statistics for orthopedic surgery 2006-2007: data from the Japanese Diagnosis Procedure Combination database. J Orthop Sci. 2010;15(2):162-70.

75. Glumac S, Kardum G, Sodic L, Supe-Domic D, Karanovic N. Effects of dexamethasone on early cognitive decline after cardiac surgery: A randomised controlled trial. Eur J Anaesthesiol. 2017:34(11):776-84.

76. Qiao H, Zhang J, Liang WM. Feasibility and safety of acute hypervolemic hemodilution in neurosurgical patients. Natl Med J Chin. 2011;91(37):2630-3.

77. Wu Y, Yang T, Zeng Y, Si H, Cao F, Shen B. Tranexamic acid reduces blood loss and transfusion requirements in primary simultaneous bilateral total knee arthroplasty: a meta-analysis of randomized controlled trials. Blood Coagul Fibrinolysis. 2017;28(7):501-8.

\section{Publisher's Note}

Springer Nature remains neutral with regard to jurisdictional claims in published maps and institutional affiliations. 